\title{
APPLICATION OF GREY SYSTEM MODEL TO THERMAL ERROR MODELING ON MACHINE TOOLS
}

\author{
Yongxiang Li', Jianguo Yang, Hongtao Zhang and Hengchao Tong \\ 'School of Mechanical Engineering, Shanghai JiaoTong University, China; Email: \\ liyongxiang@situ.edu.cn.
}

\begin{abstract}
The basic principle and method of grey system model and its application in thermal error modeling on machine tools has been presented. How to utilize thermal error sequence surveyed to model and predict is discussed by adopting total data GM $(1,1)$ model, new information $\mathrm{GM}(1,1)$ model and metabolic $\operatorname{GM}(1,1)$ model after function transform is brought forward to improve degree of smoothness of primary data sequence. Finally prediction precision of metabolic GM $(1,1)$ model is $7.2 \%$ and $15.46 \%$ higher than those of new information GM $(1,1)$ model and total data $\mathrm{GM}(1,1)$ model respectively. The comparison of the experiment's results indicates the accuracy and robustness advantages of metabolic GM $(1,1)$ model over other two models.
\end{abstract}

Key words: Machine tool, Thermal error, Metabolic GM $(1,1)$ model, Modeling

\section{INTRODUCTION}

In the precision machinery processing, machine tool thermal error is the major factor to cause workpiece's error, how to control machine tool thermal error becomes essential technique to increase their processing precision. These errors could be decreased by amending machine tools' self-structure through advanced design and manufacture technique, but with precision's improvement of machine tools, corresponding expense would be increased. Error compensation technique is regarded as a effective and economical way, it could make workpiece more precise than machine tool without improving machine tools' themselves precision. So the kind of way has already received more and more widespread value in the mechanical processing industry.

This project is supported by A Foundation for the Author of National Excellent Doctoral Dissertation of China under the grant No. 200131

Please use the following format when citing this chapter:

Li, Yongxiang, Yang, Jianguo, Zhang, Hongtao, Tong, Hengchao, 2006, in International Federation for Information Processing (IFIP), Volume 207, Knowledge Enterprise: Intelligent Strategies In Product Design, Manufacturing, and Management, eds. K. Wang, Kovacs G., Wozny M., Fang M., (Boston: Springer), pp. 511-518. 
If compensation technique is used for machine tool thermal error, three problems must be solved: first, the detection of thermal error; second, the establishment of prediction model of thermal error; third, the implementation of real-time compensation of thermal error. Establishment of prediction model is the most essential among them. For thermal error is in great degree decided by many kinds of factors such as the processing condition, the processing cycle, the use of coolant as well as the environment, moreover, machine tool thermal error presents the non-linearity and interaction, therefore, it is quite difficult to precisely establish mathematical model of thermal error with theoretical analysis only [1]. Grey system theory was founded by Professor Deng Julong [2-3] of Huazhong University of Science and Technology. For grey system model includes content of general linear regression and power function, it could make up the deficiency of traditional difference and disperse model and present a strong tendency, especially for the system with both the known information and the unknown information, grey system model has very good fitting, prediction effect. Moreover, grey system model has quick speed of modeling and computation, has not high requirement on primary data and does not depend on massive data information as modeling foundation, so grey system model is a simple and convenient model which has lower request on gathered data information [4]. The work of this article is to apply improved grey system model to modeling of machine tool thermal error, the results show that metabolic GM $(1,1)$ model is the most satisfied among three kinds of $\operatorname{GM}(1,1)$ model.

\section{GREY SYSTEM MODEL}

\subsection{Establishment of GM (1,1) model}

Assumption $\left\{x^{(0)}(i)\right\}(i=1,2, \cdots, n)$ is a primary data sequence, after once accumulating generation, it becomes a new sequence as follows:

$$
x^{(1)}(k)=\sum_{j=1}^{k} x^{(0)}(j) ; \quad k=1,2, \cdots, n
$$

Then corresponding differential equation of $\operatorname{GM}(1,1)$ model is expressed as:

$$
\frac{d x^{(1)}}{d t}+a x^{(1)}=u
$$


where $a$ is development grey number, $u$ is endogenetic control grey number. number.
$a$ is defined as estimating parameter vector, $\hat{a}=\left[\begin{array}{l}a \\ u\end{array}\right]$, according to least
square method, using following formula

$$
\hat{a}=\left(B^{T} B\right)^{-1} B^{T} y_{N}
$$

$$
\text { where } B=\left[\begin{array}{cc}
-\frac{1}{2}\left[x^{(1)}(1)+x^{(1)}(2)\right] & 1 \\
-\frac{1}{2}\left[x^{(1)}(2)+x^{(1)}(3)\right] & 1 \\
\cdots & \ldots \\
-\frac{1}{2}\left[x^{(1)}(n-1)+x^{(1)}(n)\right] & 1
\end{array}\right],
$$

to resolve differential equation (2), the solution is expressed as

$$
\hat{x}(k+1)=\left[X^{(0)}(1)-\frac{u}{a}\right] e^{-a k}+\frac{u}{a}
$$

Equation (4) is just GM $(1,1)$ and it is carried on once inverse accumulating generation, the reductive model is got by

$$
\hat{x^{(0)}}(k+1)=\hat{x^{(1)}}(k+1)-\hat{x^{(1)}}(k) \text { or } x^{\hat{(0)}}(k+1)=(-a)\left[x^{(0)}(1)-\frac{u}{a}\right] e^{-a k}
$$

\subsection{Function transform modeling of grey system}

Considering grey system model's requirement of degree of smoothness for disperse data sequence, for non-minus primary data $\left\{x^{(0)}(i)\right\}(i=1,2, \cdots, n), f(i)$, as a non-minus and monotonously degressive sequence, would be chosen to transform $x^{(0)}(i)$, a new sequence is given by: $F\left(x^{(0)}(i)\right)=x^{(0)}(i) \times f(i)$, which would evidently improve degree of smoothness of primary data $x^{(0)}(i)$; then $F\left(x^{(0)}(i)\right)$ would be modeled by grey system model and be reverted by contrary transform, finally, modeling precision of system would be heightened markedly [5]. 


\subsection{Improvement of GM $(1,1)$ model [6]}

In practical modeling, not all data of the primary data sequence is used in modeling, part of data could also be used to establish a model. Generally speaking, different data could establish different model, even if the same kind of GM $(1,1)$ model is established, the values of parameter $a, b$ are different with different selected data. This kind of instance precisely reflects the influence of different situation and condition on system characteristic in the model.

Assumption $X^{(0)}=\left(x^{(0)}(1), x^{(0)}(2), \cdots, x^{(0)}(n)\right), x^{(0)}(n)$ is chosen as origin of time axis, then $t<n$ is considered as past, $t=n$ is present and $t>n$ is future.

1. GM $(1,1)$ model, established by $X^{(0)}=\left(x^{(0)}(1), x^{(0)}(2), \cdots, x^{(0)}(n)\right)$, is called total data GM $(1,1)$ model;

2. $\mathrm{GM}(1,1)$ model, established by $X^{(0)}=\left(x^{(0)}\left(k_{0}\right), x^{(0)}\left(k_{0}+1\right), \cdots, x^{(0)}(n)\right)$, $\forall k_{0}>1$, is called partial-data $\mathrm{GM}(1,1)$ model;

3. Assumption $x^{(0)}(n+1)$ is latest information, which is placed in $X^{(0)}$, then GM $(1,1)$ model, established by $X^{(0)}=\left(x^{(0)}(1), x^{(0)}(2), \cdots, x^{(0)}(n)\right.$, $\left.x^{(0)}(n+1)\right)$ is called new information GM(1,1) model;

4. If latest information $x^{(0)}(n+1)$ is placed and the oldest information $x^{(0)}(1)$ is taken out, then $\mathrm{GM}(1,1)$ model, established by $X^{(0)}=\left(x^{(0)}(2), \cdots, x^{(0)}(n), x^{(0)}(n+1)\right)$, is called metabolic $\operatorname{GM}(1,1)$ model.

\section{APPLICATION AND ANALYSIS OF GREY SYSTEM MODEL}

\subsection{Measurement and analysis of thermal errors of machine tool}
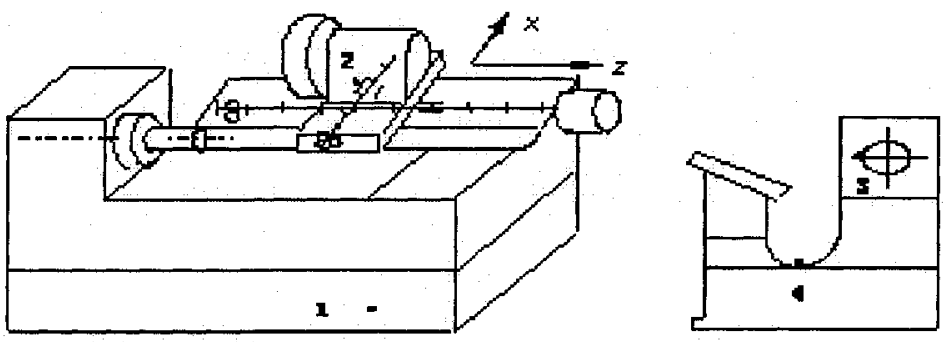

Figure. 1. Sketch map of turning center 
The method is applied to a CNC turning center, as shown in Fig. 1, and is used in study of thermal error's compensation [7]. Sketch map of thermal error's measurement is shown in Fig. 2.

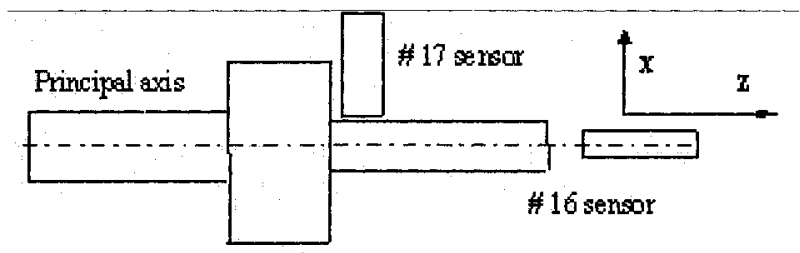

Figure. 2. Measurement of thermal errors of principal axis

Two displacement sensors fixed on knife rest measure thermal errors between principal axis of $X$ direction (17) and $Z$ direction (16) and knife rest. For workpiece is very short, the error of leaning angle is neglected.

\subsection{Prediction analysis of grey system model}

Grey prediction model is to predict self-development according to dynamic differential equation established by its own data [8]. The pretreatment of grey varible is generally accumulating or inverse accumulating primary data, in order to weaken even eliminate randomicity information of original sequence and generate data sequence with strong regularity [4]. The text chooses radial thermal errors in the process of turning as researchful objects (for workpieces' precision of radial dimension has rather high request, radial thermal error of machine tool is only considered), in practice, researchful objects are workpieces' errors of radial dimension changed along with the machine tool's temperature or the time [9]. Change figure of thermal errors of turning center is shown in Fig. 3.

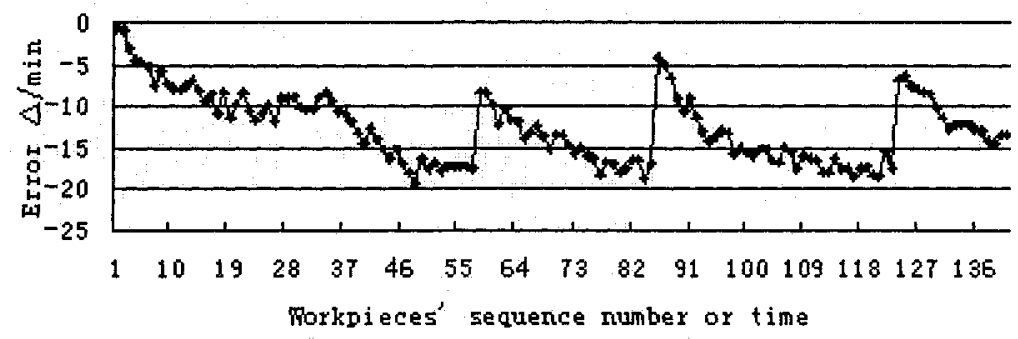

Figure. 3. Change figure of thermal errors of turning center

According to above mentioned grey system theory, the most basic GM $(1,1)$ model, namely total data GM $(1,1)$ model, is firstly used in thermal error sequence modeling of turning center. Considering the fact that grey 
system model requires that data sequence must have high degree of smoothness, first, primary data sequence, $x^{(0)}(i)(i=1,2, \cdots, n)$, would be transformed into positive data sequence by absolute value operation due to the negative values of thermal errors of machine tool shown in Fig. 3; second, positive data sequence would be transformed by a non-minus and monotonously degressive sequence $f(i)=10 / i(\mathrm{i}=1,2, \cdots, \mathrm{n})$ again, then get new data sequence, $F\left(x^{(0)}(i)\right)=x^{(0)}(i) \times f(i)$; third, the former 100 data of new data sequence are used to establish GM $(1,1)$ model according to (1)- (5); fourth, GM $(1,1)$ model would be reverted into forecasting $\operatorname{GM}(1,1)$ model by contrary function transform; fifth, forecasting GM $(1,1)$ model could forecast the latter 41 data of primary data sequence, then forecasting value of thermal error for machine tool would be obtained, compared with primary data sequence, absolute error and relative error between forecasting values and practical values are also obtained. Finally, both new information GM $(1,1)$ model and metabolic GM $(1,1)$ model are also used to model and predict thermal error sequence.

The comparisons of predicted value and practical value of all models are drawn in Fig. 4-Fig. 6 according to above predicted results. It can be seen from 3 figures clearly, prediction precision of metabolic GM $(1,1)$ model is highest, new information GM $(1,1)$ model is next, total data GM $(1,1)$ model is worst. Prediction precision of metabolic GM $(1,1)$ model is $7.2 \%$ and $15.46 \%$ higher than those of new information GM $(1,1)$ model and total data GM $(1,1)$ model respectively. Thermal error of turning center can be kept in $7.58 \mu \mathrm{m}$ from primary about $20 \mu \mathrm{m}$ through error compensation using metabolic GM $(1,1)$ model. The reason was that along with passing time, some random vibration or driving factor would enter into the system constantly in the developing course of any grey system, and then affect systemic development, however, metabolic GM $(1,1)$ model just renews new information constantly and removes old information duly, so that modeling sequence could reflect systemic present characteristic and obtain higher prediction precision. The comparison of error curve of three grey prediction models is shown in Fig. 7.

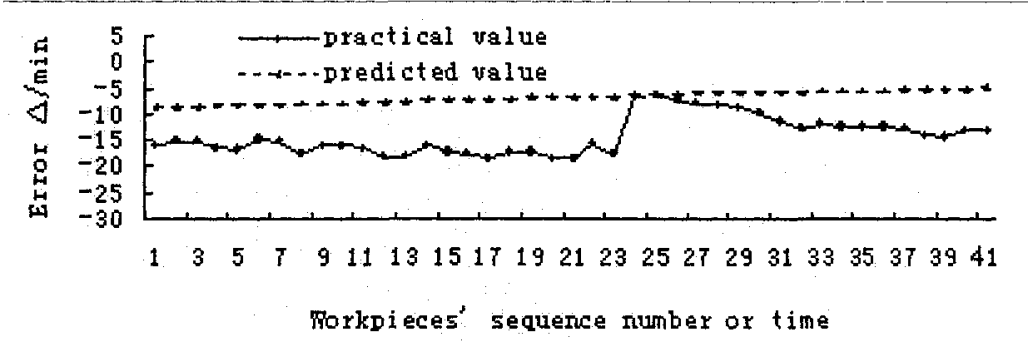

Figure. 4. Comparison of predicted curve and practical curve of total data $\operatorname{GM}(1,1)$ model 


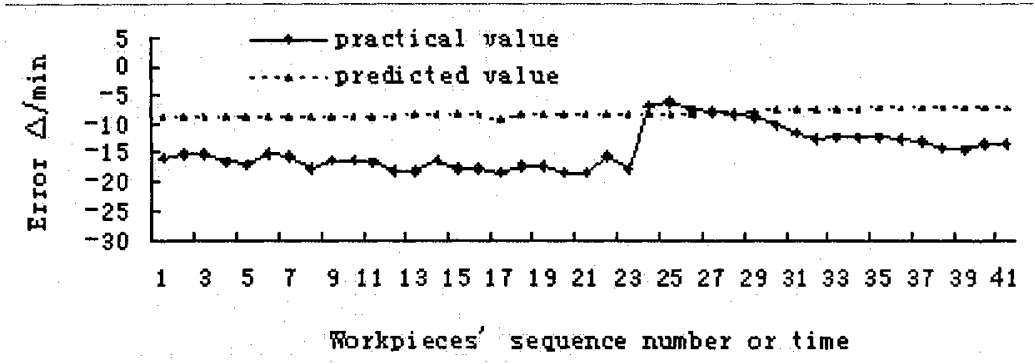

Figure. 5. Comparison of predicted curve and practical curve of new information GM $(1,1)$ model

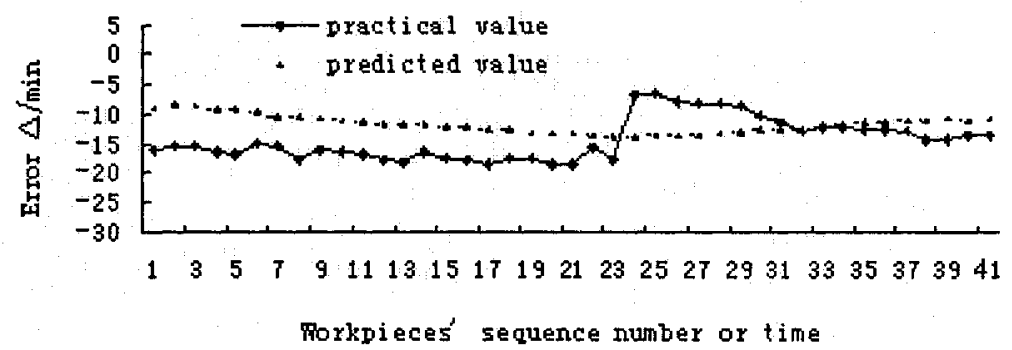

Figure. 6. Comparison of predicted curve and practical curve of metabolic GM $(1,1)$ model

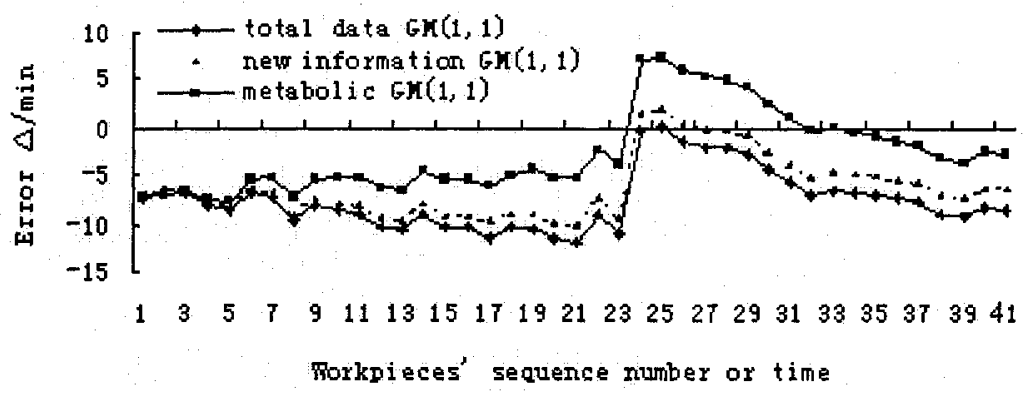

Figure. 7. Comparison of errors curve of three kinds of GM $(1,1)$ model

\section{CONCLUSION}

Grey system model, a dynamic model, weakens randomicity and shows systemic assured developing trendency [10]. The text just regards machine 
tool thermal error as a grey variable according to generation mechanism and anfractuosity of machine tool thermal error, and adopts grey system model to model. At the same time, after degree of smoothness of primary data sequence is improved by function transform, based on total data GM $(1,1)$ model, the text brings forward new information GM $(1,1)$ model and metabolic GM $(1,1)$ model to model for machine tool thermal error.

It could be seen that both new information GM $(1,1)$ model and metabolic GM $(1,1)$ model have higher predicted precision than total data GM $(1,1)$ model from predicting result of machine tool thermal error, metabolic GM $(1,1)$ model is optimal model among three kinds of GM $(1,1)$ model, for the function of the old data would decrease with the development of system, it is obviously reasonable to remove those old data which could not reflect systemic present characteristics, moreover, metabolic GM $(1,1)$ model could avoid the difficult caused by continual increase of computing quantity with the increase of information quantity.

\section{REFERENCES}

1. Y. Q. Ren, (2004), Applied research on high-efficiency measurement, modeling and compensation for errors on NC machine tools, Shanghai: Shanghai JiaoTong University.

2. J. L. Deng, (1989), Introduction to grey system theory, The Journal of Grey System, vol. 1, pp. 1-24.

3. J. L. Deng, (2002), Basis of grey theory, Wuhan: Huazhong University of Science and Technology Press.

4. Y. L. Pan, S. X. Zheng, (2002), Improvement for the precision of instruments by a grey system model, Cryogenics and Superconductivity, vol. 30, pp. 65-67.

5. Y. X. Luo, L. T. Zhang and M. Li, (2001), Grey system theory and application in the mechanical engineering, Changsha: National University of Defense Technology Press.

6. S. F. Liu, T. B. Guo and Y. G. Dang, (1999), Grey system theory \& Application, Beijing: Science Press.

7. J. G. Yang, J. X. Yuan and J. Ni, (1999), Thermal error mode analysis and robust modeling for error compensation on a CNC turning center, International Journal of Machine Tools and Manufacture, vol. 39, pp. 1367-1381.

8. Z. T. Wu, Y. Li and Q. Li, (1998), Grey prediction of running status trendency of complex mechanical equipment, Mechanical Research \& Application, vol. 1, pp. 44-45.

9. J. G. Yang, (1998), Error synthetic compensation technique and application for NC machine tools, Shanghai: Shanghai JiaoTong University.

10. P. Gong, Y. T. Fei, (2001), Grey evaluation method of dynamic measurement errors based on error propagation theory, Tool Engineering, vol. 5, pp. 31-34. 